DAMTP-1999-101

\title{
Non-BPS D8-branes and Dynamic Domain Walls in Massive IIA Supergravities
}

\author{
A. Chamblin*, M.J. Perry ${ }^{\dagger}$ and H.S. Reall ${ }^{\ddagger}$ \\ University of Cambridge \\ DAMTP \\ Silver Street \\ Cambridge, CBз 9EW \\ United Kingdom
}

(5 August 1999)

\begin{abstract}
We study the D8-branes of the Romans massive IIA supergravity theory using the coupled supergravity and worldvolume actions. D8 branes can be regarded as domain walls with the jump in the extrinsic curvature at the brane given by the Israel matching conditions. We examine the restrictions that these conditions place on extreme and non-extreme solutions and find that they rule out some of the supersymmetric solutions given by Bergshoeff et al. We consider what happens when the dilaton varies on the worldvolume of the brane, which implies that the brane is no longer static. We obtain a family of D8-brane solutions parametrized by a non-extremality term on each side of the brane and the asymptotic values of the 10-form field. The nonextremality parameters can be related to the velocity of the brane. We also study 8-brane solutions of a massive IIA supergravity theory introduced by Howe, Lambert and West. This theory also admits a 10-form formulation, but the 10-form is not a R-R sector field and so these 8-branes are not D-branes.
\end{abstract}

11.10.Lm, 97.60.Lf, 04.20.Jb, 11.25.Hf, 11.30.Pb

Typeset using REVTEX

\footnotetext{
*H.A.Chamblin@damtp.cam.ac.uk

${ }^{\dagger}$ malcolm@damtp.cam.ac.uk

${ }^{\ddagger}$ H.S.Reall@damtp.cam.ac.uk
} 


\section{INTRODUCTION}

Perhaps the most important thing which string duality teaches us is that in order to have a consistent string theory we have to include objects known as "D-branes". These D-branes, which are just hyperplanes where open strings are allowed to end, were shown by Polchinski [1] to be the carriers of ten dimensional Ramond-Ramond (R-R) charge. T-duality requires the existence of D-branes [2] and, once stringy effects are taken into account, the D-branes become dynamical objects. Indeed, the collective coordinates for the transverse fluctuations of a D-brane are just the massless open string excitations that live on the brane worldvolume.

Once one recognizes the existence of D-branes, it is natural to start asking questions about their gross kinematical and/or dynamical properties. For example, is there any constraint on the topology of these branes? What happens when we push a brane away from extremality? Can these branes rip, or tear, by some semi-classical decay process analagous to the decay of cosmic strings (by black hole pair creation)?

Before proceeding with the principal construction, it is useful if we first recall some basic facts about D-branes [3]. To begin, let us focus just on the bosonic terms in the effective field theory actions which arise from the IIA and IIB string theories. These field theories are of course just the type IIA and type IIB supergravity theories. In the Neveu-Schwarz-NeveuSchwarz(NS-NS) sector these two theories have identical field content, consisting of a metric tensor $g_{i j}$, an antisymmetric rank two tensor potential $b_{i j}$ and a scalar dilaton field $\phi$. On the other hand, in the Ramond-Ramond (R-R) sector the field content of the two theories is quite different. Explicitly, the bosonic sector for the effective action of IIA supergravity is given as"

$$
\begin{aligned}
S_{I I A}= & \frac{1}{2 \kappa^{2}} \int d^{10} x \sqrt{-g}\left[e ^ { - 2 \phi } \left(R+4(\partial \phi)^{2}\right.\right. \\
& \left.\left.-\frac{1}{2 \cdot 3 !} H^{2}\right)-\left(\frac{1}{2 \cdot 2 !} F_{(2)}{ }^{2}+\frac{1}{2 \cdot 4 !} F_{(4)}^{2}\right)\right] \\
& -\frac{1}{4 \kappa^{2}} \int F_{(4)} \wedge F_{(4)} \wedge b,
\end{aligned}
$$

whereas the effective action for IIB supergravity takes the form

$$
\begin{aligned}
S_{I I B}= & \frac{1}{2 \kappa^{2}} \int d^{10} x \sqrt{-g}\left[e ^ { - 2 \phi } \left(R+4(\partial \phi)^{2}\right.\right. \\
& \left.-\frac{1}{2 \cdot 3 !} H^{2}\right)-\frac{1}{2}\left(\partial A_{(0)}\right)^{2} \\
& \left.-\frac{1}{2 \cdot 3 !}\left(A_{(0)} B+F_{(3)}\right)^{2}\right]
\end{aligned}
$$

(where we have omitted terms involving the self-dual five-form which cannot be expressed in a covariant fashion). Here, $F_{(n)}=n d A_{(n-1)}$ are the antisymmetric $n$-form R-R field strengths, with $(n-1)$-form potentials $A_{(n-1)}$, and $H=3 d b$ is the antisymmetric NS-NS

\footnotetext{
${ }^{1}$ We use a positive signature metric and the curvature convention for which de Sitter space has positive Ricci scalar.
} 
three-form field strength. The string coupling $g_{s}$ is given in terms of the dilaton by the relation $g_{s}=e^{\phi}$. Thus, in both of the above actions the NS-NS sector is multiplied by a factor of $g_{s}{ }^{-2}$; this means that NS-NS effects arise in ordinary string perturbation theory. On the other hand, the R-R terms are not multiplied by any such factor and so we know that fundamental string states do not carry R-R gauge charges. As we have already mentioned, the mystery of the R-R gauge fields was resolved when it was shown that R-R charge was carried by the topological defects known as D-branes [1].

These actions do not tell the whole story about D-brane configurations. This is because they really only tell us about what life is like for massless closed string states which exist "off" the D-branes and how these states couple to the D-branes. For this reason, the supergravity effective actions are called the "bulk" terms. There should also be an effective description of life on the brane worldvolume. Indeed, such an effective world-volume action (denoted $S_{W V}$ ) does exist and it is characterized by a $\mathrm{D}(\mathrm{p})$-brane tension, $\mu_{p}$, and R-R charge density form $f_{p}$ as shown below:

$$
S_{W V}=-\mu_{p} \int d^{p+1} \sigma e^{-\phi} \sqrt{\operatorname{det}\left(h_{\mu \nu}+b_{\mu \nu}+2 \pi \alpha^{\prime} F_{\mu \nu}\right)}-f_{(p)} \int d^{p+1} \sigma A_{(p+1)}
$$

Here, $\sigma$ denotes the coordinates tangent to the brane worldvolume, $h_{\mu \nu}, b_{\mu \nu}$ and $\phi$ are the pullback of the (respective) spacetime fields to the brane worldvolume. $F_{\mu \nu}$ is an ordinary Maxwell gauge field which lives on the brane worldvolume. Again, $A_{(p+1)}$ is an antisymmetric $(p+1)$-form R-R potential which couples naturally to the p-brane worldvolume (hence the appearance of the R-R charge density $\left.f_{(p)}\right)$. This form of the action is very useful if one is interested in studying "light" branes, which have negligible gravitational fields (so that one can ignore the bulk supergravity contributions). In this limit a beautiful picture emerges, in which fundamental strings ending on a D-brane appear, from the point of view of the brane worldvolume theory, as Coulomb-like point particle solutions of the Born-Infeld theory on the brane (Gibbons refers to all such solitonic configurations in Born-Infeld theory as "BIons") [4.5]. Similarly, one can also think of an M2 brane ending on an M5 brane as a "vortex"-type BIon living on the M5 brane worldvolume, and so on. While these are certainly beautiful and promising results, it is still important to understand how strings and branes in general will interact when they are heavy i.e. the effects of gravity are taken into account. Presumably, addressing this problem will have to involve somehow writing down an effective action which interpolates between the world-volume and bulk terms as gravity is turned up. In this paper, we consider the simpler problem of how the world-volume and bulk terms interact with each other when the branes gravitate.

We concentrate on the case of D8-branes of massive IIA supergravity because we can then use the theory developed in [6] to solve the equations of motion of the coupled worldvolumebulk system for moving branes. D8-branes can be viewed as domain walls, which have been extensively studied in cosmology and supergravity. The gravitational effects of a domain wall are described by the Israel equations which are discussed in section [1]. The massive IIA supergravity theory and its D8 branes are described in $[1]$. In section $\mathbb{D}$ we give our solutions describing moving D8 branes. Section $\nabla$ describes dynamic branes of a different massive IIA supergravity and we conclude in section $\nabla$. 


\section{THE ISRAEL MATCHING CONDITIONS AND DYNAMIC DILATONIC DOMAIN WALLS}

The fact that 8-branes divide ten-dimensional spacetime up into domains is reminiscent of domain walls in cosmology. As is well known, various cosmological models assume that a variety of phase transitions took place in the early universe. In such transitions, symmetries which are only valid at high temperatures are broken as the universe cools down. Bubbles of the new phase are nucleated in regions of the old phase and may expand; if the rate of production of these bubbles is not diluted by the rate of expansion of the universe, the process of bubble nucleation, expansion and amalgamation will continue until the universe is filled with new phase (with perhaps a few bubbles of the old phase left over). When the new phase fills the entire universe, the transition is said to be complete.

The dynamical evolution of these bubbles when the effects of gravity are included, has been studied by a number of authors [7, 8]. These studies involve understanding the Einstein equations when the source is a thin shell, or domain wall. In such situations, the spacetime has low differentiability and one has to regard the curvature as a distribution. It was shown long ago [9] that the correct formalism for studying such a problem involves constructing metric junction conditions for the thin shells. These junction conditions, commonly referred to as the "Israel matching conditions", state the discontinuity in the extrinsic curvature of the shell is related to the energy-momentum tensor $t_{a b}$ of the matter on the shell by

$$
\left[K_{a b}-K h_{a b}\right]=8 \pi G t_{a b}
$$

where $K$ denotes the trace of the extrinsic curvature $K_{a b}$ and $h_{a b}$ is the induced metric on the shell. A simple derivation of this equation is given in [6]. Thus the energy momentum of an idealized domain wall gives rise to a jump in the extrinsic curvature of surfaces parallel to the wall as one moves through the wall. These conditions are easily satisfied when the only bulk energy density comes from a cosmological constant and the energy density of the wall is constant. In supergravity theories one typically has scalar fields present that couple to domain walls and until recently solutions had only been found when these fields are constant over the worldvolume of the wall [10 [12]. A method for dealing with the case of non-constant worldvolume fields was given in [6]. If one assumes that the bulk on both sides of the wall is static then consistency of the Israel conditions yields non-trivial relationships between the metric in the bulk and the matter on the wall. These can be used to solve the Einstein equations in the bulk. The resulting spacetime usually contains cosmological horizons so, although one starts from a static ansatz, the spacetime is actually time-dependent. We will use these results when we describe the dynamic branes of the Romans theory because there the dilaton has a Liouville potential and therefore has to run in the bulk.

\section{D8 BRANES AND THE ROMANS THEORY}

The D8-brane of IIA string theory couples to the ten-form field strength $F_{10}$ of the R-R sector. As Polchinski [1] pointed out, this ten-form is not a dynamical variable; rather, it is just a constant field which generates a uniform physical energy density which permeates space. This energy density is proportional to the square of the mass term of the massive IIA 
supergravity theory derived by Romans [13]. In this theory, the mass arises from a Higgs mechanism in which the two-form "eats" the vector.

The ten form formulation of the Romans massive IIA supergravity was given by Bergshoeff et al. [14]. For our purposes the relevant part of the action in the string frame is

$$
S=\frac{1}{2 \kappa^{2}} \int d^{10} x \sqrt{-g}\left(e^{-2 \phi}\left(R+4(\partial \phi)^{2}\right)-\frac{1}{2} M^{2}\right)+\frac{1}{2 \kappa^{2}} \int \frac{1}{10} M F_{(10)},
$$

where $M$ is an auxiliary field which can be eliminated via its equation of motion. The Einstein frame metric is given by $g_{a b}^{(E)}=e^{-\phi / 2} g_{a b}^{(S)}$. In this frame the action is

$$
S=\frac{1}{2 \kappa^{2}} \int d^{10} x \sqrt{-g}\left(R-\frac{1}{2}(\partial \phi)^{2}-\frac{1}{2} e^{5 \phi / 2} M^{2}\right)+\frac{1}{2 \kappa^{2}} \int \frac{1}{10} M F_{(10)} .
$$

We shall set $\kappa=1$. The ten form is given in terms of its nine form potential by $F_{(10)}=$ $10 d A_{(9)}$. Varying $A_{(9)}$ gives $M=$ const. and varying $M$ gives $F_{(10)}=10 M e^{5 \phi / 2} \eta_{(10)}$ where $\eta_{(10)}$ is the volume form of the spacetime. Note that varying the metric does not affect the final term. Therefore the Einstein equations are the same as those for which the only matter is a scalar field $\phi$ with potential

$$
V(\phi)=\frac{1}{4} M^{2} e^{5 \phi / 2}
$$

The relevant part of the D8 brane string frame action is [3]

$$
S_{\text {brane }}=-\mu \int d^{9} \xi \sqrt{-h} e^{-\phi} \pm \mu \int A_{(9)}
$$

where $h$ is determinant of the induced metric on the brane world volume and the final term involves the pull back of the bulk nine form potential to the brane's world volume. The upper sign refers to a brane and the lower sign to an anti-brane. In the Einstein frame the action is

$$
S_{\text {brane }}=-\int d^{9} \xi \sqrt{-h} \hat{V}(\phi) \pm \mu \int A_{(9)}
$$

where

$$
\hat{V}(\phi)=\mu e^{5 \phi / 4}
$$

Note that the final (Wess-Zumino) term is independent of the metric so it does not contribute to the world volume energy momentum of the brane and therefore does not occur in the Israel equations. However the brane is a source for the ten form so we expect $F_{(10)}$ to have a discontinuity at the brane arising from this term. To see this, vary $A_{(9)}$ in the total action $S_{\text {bulk }}+S_{\text {brane }}$ to obtain the surface term

$$
\frac{1}{2} \int([M] \pm 2 \mu) \delta A_{(9)}
$$


where we refer to the bulk regions on the two sides of the brane as $(+)$ and $(-)$ and $[M] \equiv[M]_{-}^{+}$denotes the discontinuity of $M$ at the brane ${ }^{2}$. If this term is to vanish then we need

$$
[M]=\mp 2 \mu,
$$

which relates the jump in the field strength to the charge of the brane.

There will also be a discontinuity in the normal derivative of the dilaton at the brane. Varying the dilaton gives a surface term

$$
-\int d^{9} \xi \sqrt{-h}\left(\frac{1}{2}[n . \partial \phi]+\frac{d \hat{V}}{d \phi}\right) \delta \phi,
$$

where $n$ is the unit normal pointing away from the $(+)$ region of the bulk. The surface term vanishes for arbitrary $\delta \phi$ only if

$$
[n . \partial \phi]=-2 \frac{d \hat{V}}{d \phi}
$$

This equation ensures that energy-momentum is conserved as the brane moves through regions of varying $\phi[6]$.

\section{DYNAMIC D8-BRANES OF THE ROMANS THEORY}

We can apply the method developed in [6] to solve the equations of motion of the coupled brane-bulk system. The starting point is the assumption that the metric is static on both sides of the brane. It will turn out that this assumption is less restrictive than it may seem because the bulk spacetime typically contains cosmological horizons beyond which it is manifestly time dependent. We shall assume that on each side the bulk metric can be written

$$
d s^{2}=-U_{ \pm}\left(r_{ \pm}\right) d t_{ \pm}^{2}+U_{ \pm}\left(r_{ \pm}\right)^{-1} d r_{ \pm}^{2}+R_{ \pm}\left(r_{ \pm}\right)^{2} d \Omega_{k}^{2}
$$

where $d \Omega_{k}^{2}$ is the line element on an eight dimensional Einstein space with metric $\bar{g}_{m n}$ and Ricci tensor $\bar{R}_{m n}=7 k \bar{g}_{m n}$ with $k \in\{-1,0,1\}$. The dilaton is assumed to be a function only of $r_{ \pm}$. The bulk field equations (i.e. the Einstein equations and the dilaton equation of motion) for this ansatz were given in 60. Let the brane position be $t_{ \pm}=t_{ \pm}(\tau), r_{ \pm}=r_{ \pm}(\tau)$

\footnotetext{
2 The signs are determined as follows. Choose an oriented atlas for the whole spacetime (including the brane) and consider a chart $x^{a}$ defined in a neighbourhood of the brane such that the brane is at $x^{9}=0$. Let $(+)$ denote the region $x^{9}<0,(-)$ the region $x^{9}>0$ and $n$ the outward unit normal to the $(+)$ region. The volume form of the bulk at the brane is $\eta_{(9)} \wedge n$ where $\eta_{(9)}$ is the volume form of the brane world volume.

${ }^{3}$ The equations in [6] use the rescaled dilaton $\phi / \sqrt{2}$.
} 
where $\tau$ denotes proper time of an observer at rest relative to the spatial sections of the brane i.e. the induced metric on the brane take the Friedmann-Robertson-Walker form

$$
d s^{2}=-d \tau^{2}+R(\tau) d \Omega_{k}^{2}
$$

Continuity of the metric at the brane requires that $R_{+}\left(r_{+}(\tau)\right)=R_{-}\left(r_{-}(\tau)\right)=R(\tau)$. Henceforth we shall drop the \pm subscripts except where this might cause confusion.

The proper velocity of the brane is

$$
u^{a}=\left(\sigma U^{-1} E, \frac{d r}{d \tau}, 0, \ldots, 0\right),
$$

where $\sigma=+1$ when $U(r)>0$ i.e. when $t$ is a time coordinate. If $t$ becomes a spatial coordinate (e.g. after crossing a horizon) then $\sigma= \pm 1$. The quantity $E$ is the energy per unit mass of a particle comoving with the brane

$$
E=\sqrt{U+\left(\frac{d r}{d \tau}\right)^{2}}
$$

The unit normal is

$$
n_{a}=\epsilon\left(\sigma \frac{d r}{d \tau},-U^{-1} E, 0, \ldots, 0\right)
$$

where $\epsilon= \pm 1$ is chosen so that the normal points out of the $(+)$ region and into the $(-)$ region.

The extrinsic curvature $K_{a b} \equiv h_{a}^{c} h_{b}^{d} \nabla_{c} n_{d}$ is most easily computed in the basis $u^{a}, n^{a}, e_{(1)}^{a}, \ldots, e_{(8)}^{a}$, where the $e_{(i)}^{a}$ are a basis for the spatial sections $t=$ const., $r=$ const. See [6] for the details of this calculation. The result is

$$
\begin{gathered}
K_{00}=\epsilon \frac{d E}{d \tau} / \frac{d r}{d \tau}, \\
K_{i j}=-\epsilon E \frac{R^{\prime}}{R} h_{i j},
\end{gathered}
$$

where a prime denotes a derivative with respect to $r$. The Israel equations 2.1 relate the jump in the extrinsic curvature to the brane world volume energy momentum tensor,

$$
t_{a b}=-\hat{V}(\phi) h_{a b} .
$$

They can be rewritten as

$$
\left[K_{a b}\right]=\frac{\hat{V}(\phi)}{8} h_{a b}
$$

so the $i j$ components reduce to

$$
\left[\epsilon E \frac{R^{\prime}}{R}\right]=-\frac{1}{8} \hat{V}(\phi) .
$$


Note that $\epsilon_{+}$and $\epsilon_{-}$need not be the same. Equation 3.10 gives the discontinuity in the normal derivative of $\phi$

$$
\left[\epsilon E \phi^{\prime}\right]=2 \frac{d \hat{V}}{d \phi} .
$$

The similarity of these two equations allows us to relate $\phi$ and $R$. Note that $d \phi / d R$ must be continuous across the brane and that

$$
\phi_{ \pm}^{\prime}=\frac{d \phi}{d R} R_{ \pm}^{\prime}
$$

This can be substituted into equation 4.11 and compared with equations 4.10 to yield

$$
\frac{d \phi}{d R}=-\frac{16}{R \hat{V}} \frac{d \hat{V}}{d \phi}
$$

This equation has to hold at every point visited by the brane so if the brane moves through a range of $R$ then it can be integrated to yield $\phi$ as function of $R$.

We now turn to the 00 component of the Israel conditions, which can be written

$$
\frac{d}{d \tau}\left[\epsilon E \frac{R^{\prime}}{R}\right]-\frac{1}{R} \frac{d R}{d \tau}\left[\epsilon E \frac{R^{\prime}}{R} X\right]=-\frac{\hat{V}}{8 R} \frac{d R}{d \tau},
$$

where

$$
X \equiv\left(\frac{R^{\prime}}{R}\right)^{\prime}\left(\frac{R^{\prime}}{R}\right)^{-2} .
$$

In [6] the bulk spacetime was assumed to be symmetric under reflection in the brane. Consistency of the 00 and $i j$ components of the Israel equations was then shown to reduce to a condition relating $R^{\prime}$ and $\hat{V}$. This argument does not appear possible here without appealing to the bulk field equations, which is what we shall now do. One of the Einstein equations can be written [6]

$$
\frac{R^{\prime \prime}}{R}=-\frac{1}{16} \phi^{\prime 2}
$$

Combining this with equation 4.13 and integrating gives

$$
R_{ \pm}^{\prime}\left(r_{ \pm}\right)=C_{ \pm} \hat{V}\left(\phi\left(r_{ \pm}\right)\right)
$$

where $C_{ \pm}$are constants of integration. Equations 4.13 and 4.17 relate the bulk metric and dilaton. It is easily verified that these, together with equation 4.10 ensure that equation 4.14 is satisfied.

\footnotetext{
${ }^{4}$ We would need to show that $X$ is continuous across the brane, allowing us to take it outside the square brackets in equation 4.14 and then use equation 4.10 to eliminate the square brackets. However it does not appear possible to show that $X$ must take the same value on both sides of the brane without using the bulk field equations
} 
Substituting in the explicit form for $\hat{V}$ given by equation 3.6 allows one to solve for $R_{ \pm}\left(r_{ \pm}\right)$and $\phi_{ \pm}\left(r_{ \pm}\right)$. These can then be substituted into the bulk field equations to solve for $U_{ \pm}\left(r_{ \pm}\right)$, as described in [6]. Solutions only exist when $k=0$ (i.e. for Ricci flat spatial sections[). The bulk solution takes the same form on both sides of the brane:

$$
\begin{gathered}
U_{ \pm}\left(r_{ \pm}\right)=26^{2} r_{ \pm}^{1 / 13}\left(2^{-8} M_{ \pm}^{2} e^{5 \phi_{0 \pm} / 2}-2 m_{ \pm} r_{ \pm}^{8 / 13}\right), \\
R_{ \pm}\left(r_{ \pm}\right)=r_{ \pm}^{1 / 26} \\
\phi_{ \pm}\left(r_{ \pm}\right)=\phi_{0 \pm}-\frac{10}{13} \log r_{ \pm}
\end{gathered}
$$

where $\phi_{0 \pm}$ and $m_{ \pm}$are constants of integration. Continuity of the metric at the brane requires $R_{+}\left(r_{+}(\tau)\right)=R_{-}\left(r_{-}(\tau)\right)$ hence $r_{+}(\tau)=r_{-}(\tau)$. Continuity of the dilaton at the brane then gives $\phi_{0+}=\phi_{0-}$. We can now drop the \pm subscripts when referring to $R(r), r(\tau)$ or $\phi(r)$.

By rescaling the coordinates it is possible to either set $\phi_{0}=0$ or $\left|m_{ \pm}\right|=1$ whilst preserving the form for the metric in equation 4.1, so the solutions form a 1-parameter family. However continuity of the metric of the spatial sections does not permit independent rescalings on both sides of the brane so it is not in general possible to set $\left|m_{+}\right|=\left|m_{-}\right|=1$. We shall rescale so that $\phi_{0}=0$. We shall refer to the maximal analytic extension of this solution as the maximal bulk solution to distinguish it from the solution when the jump in extrinsic curvature at the brane is taken account of.

The maximal bulk solution is singular at $r=0$. The singularity is timelike. If $m_{ \pm}$is positive then there is a cosmological horizon beyond which $r_{ \pm}$becomes a time coordinate and the metric non-static. However, as discussed in [6], our solutions remain valid when the brane crosses a horizon. If $m_{ \pm}$is negative then the solution is static everywhere. When $m_{ \pm}=0$ then we recover the supersymmetric solution of Bergshoeff et al. [14] provided we take the 8 dimensional spatial sections to be flat?. We have therefore found a oneparameter family of non-extremal generalizations of this supersymmetric solution. Note that the asymptotic (large $r$ ) behaviour of the metric is determined by the term involving $m_{ \pm}$so the non-extremal and supersymmetric solutions have different asymptotic behaviour. It is therefore not possible to define an energy for the spacetime relative to the extreme solution by using a surface integral at infinity. However $m_{ \pm}$is clearly analagous to a mass for the spacetime, so for some of our discussion we shall assume that it is positive. If $M_{ \pm}$ also vanishes (so the 10-form vanishes) then our solution becomes degenerate $(U=0)$. However it is easy to see that in this case a solution is $U=1$ with $\phi$ and $R$ constant. If

${ }^{5}$ In the nomenclature of $[6]$ these would be described as type II solutions.

${ }^{6}$ More general supersymmetric solutions have recently been discussed in [15], where it was shown that some supersymmetry will be preserved if the brane worldvolume is taken to be any Ricci flat space admitting a Killing spinor. 
the 8 dimensional spatial sections (with line element $d \Omega_{0}^{2}$ ) are flat space then this solution is simply Minkowski space.

By squaring to eliminate the square roots (in $E$ ) we obtain the brane's equation of motion

$$
\frac{1}{2}\left(\frac{d R}{d \tau}\right)^{2}+F(R)=0
$$

where

$$
\begin{aligned}
F(R) & =-\frac{32}{\mu^{2}}[m]^{2} R^{-16}-\left(\frac{1}{2}\{m\} \pm \frac{1}{4 \mu}[m]\{M\}\right) R^{-32} \\
& =-\frac{32}{\mu^{2}}\left(m_{+}-m_{-}\right)^{2} R^{-16} \mp \frac{1}{2 \mu}\left(m_{+} M_{-}-m_{-} M_{+}\right) R^{-32}
\end{aligned}
$$

The curly brackets denote a sum over the sides of the wall (i.e. $\{m\}=m_{+}+m_{-}$) and we have used equation 3.8 describing the discontinuity in $M$ at the brane. The equation of motion is the same as for a particle of unit mass and zero total energy moving in a potential $F(R)$. The fact that the same coefficient $\mu$ occurs in the Born-Infeld and Wess-Zumino terms of the D8-brane action makes the coefficient of a possible $R^{-8}$ term in $F(R)$ vanish. Specifying $r, d r / d t_{+}$and $d r / d t_{-}$at a given instant allows one to determine $m_{+}$and $m_{-}$ using this equation of motion. In this sense, initial data determines the spacetime geometry.

Note that the only dependence of $F(R)$ on $M_{ \pm}$is through the "interaction" term $[m]\{M\}$. If $[m]=0$ then the brane does not notice the jump in $M$. This is obvious on physical grounds since the brane is the source for the jump in $M$ and will not move under the influence of its own field.

The equation of motion was obtained by squaring equation 4.10. We must now go back and check which solutions of the equation of motion are still solutions of the unsquared equation. This can be written

$$
\epsilon_{+}\left|\mu^{2}-Y(R)\right|-\epsilon_{-}\left|\mu^{2}+Y(R)\right|=-2 \mu^{2},
$$

where

$$
Y(R) \equiv 2^{7}[m] R^{16} \pm \frac{1}{2} \mu\{M\}
$$

(The upper sign is for a brane and the lower sign for an anti-brane.) There are three possible solutions to this equation:

$$
\begin{aligned}
Y(R)>\mu^{2} \Rightarrow \epsilon_{+} & =+1, & & \epsilon_{-}=+1, \\
|Y(R)|<\mu^{2} \Rightarrow \epsilon_{+} & =-1, & \epsilon_{-} & =+1, \\
Y(R)<-\mu^{2} \Rightarrow \epsilon_{+} & =-1, & \epsilon_{-} & =-1 .
\end{aligned}
$$

Note that since $R$ can vary, it is possible for $\epsilon_{ \pm}$to change sign as the brane moves. This can happen only when $E_{ \pm}$vanishes, which is only possible if $U_{ \pm}(r(\tau))$ is negative i.e. $t_{ \pm}$is a spatial coordinate. Vanishing $E_{ \pm}$corresponds to $d t / d \tau=0$ which can happen if there is 
a change in the direction of motion of the brane relative to the bulk spatial sections. The geometrical interpretation of $\epsilon$ follows from

$$
-n \cdot \partial R=\epsilon_{ \pm} E_{ \pm} R^{\prime}
$$

The left hand side describes how $R$ varies as one approaches the brane from the side into which $n$ points (i.e. the $(-)$ side) in a direction perpendicular to the brane. Hence if $\epsilon_{-}=+1$ then $R$ increases towards the brane on the $(-)$ side. If $\epsilon_{-}=-1$ then $R$ decreases towards the brane on the $(-)$ side. On the other side, if $\epsilon_{+}=+1$ then $R$ decreases towards the brane on the $(+)$ side (since $n$ points into the $(-)$ side) and if $\epsilon_{+}=-1$ then $R$ increases towards the brane on the $(+)$ side. This information tells us which portions of the maximal bulk solution are relevant on each side of the wall. For example if $\epsilon_{-}=+1$ then we would take the maximal bulk solution, cut it along the brane at $r=r(\tau), t=t(\tau)$ and keep the part for which $R$ increased towards the brane in a perpendicular direction. This would give us the $(-)$ bulk region. A similar construction would give the $(+)$ region and the two are glued together at the brane.

Note that it is not possible to have $\epsilon_{+}=+1, \epsilon_{-}=-1$ which corresponds to $R$ decreasing towards the brane in the perpendicular direction on both sides. This would require a brane of negative tension.

The following subsections discuss the behaviour of the brane in several cases of interest.

\section{A. Static solutions}

The simplest case to consider is when the brane is static, by which we mean $R(\tau)$ is constant. Our method does not necessarily yield all of the solutions in this case since our bulk solutions were derived by assuming that the brane swept out a range of $r$ in the bulk. However if we assume that the bulk solution on each side of the brane is part of the maximal solution found above then we can find static solutions. Our analysis needs modifying in the static case. The reason for this is the $d r / d \tau$ in the denominator of the 00 component of the extrinsic curvature, given by equation 4.6. If this term vanishes then we must use

$$
K_{00}=\frac{\epsilon U^{\prime}}{2 \sqrt{U}},
$$

which can be obtained from equation 4.6 by taking the limit as $d r / d \tau \rightarrow 0$. Subtracting the ij component of the Israel equation from the 00 equation yields

$$
\left[\frac{\epsilon}{\sqrt{U}}\left(U^{\prime}-2 \frac{R^{\prime}}{R} U\right)\right]=0 .
$$

Substituting in the expressions for $U_{ \pm}(r)$ and $R(r)$ then leads to

$$
\frac{\epsilon_{+} m_{+}}{\sqrt{U_{+}}}=\frac{\epsilon_{-} m_{-}}{\sqrt{U_{-}}}
$$

The $i j$ component of the Israel equation becomes

$$
\epsilon_{+} \sqrt{U_{+}}-\epsilon_{-} \sqrt{U_{-}}=-\frac{13 \mu R}{4} .
$$


(We choose to work with this directly, rather than with the rearranged form involving $F(R)$ derived above.) These equations can be solved to give

$$
\sqrt{U_{ \pm}}=\mp \frac{13 \epsilon_{ \pm} \mu R}{4\left(1-m_{\mp} / m_{ \pm}\right)},
$$

from which it follows that $m_{+} \neq m_{-}$. Assume that $m_{+}>m_{-}$. It follows that $\epsilon_{+}=\epsilon_{-}=-1$. Solving for $R$ yields

$$
R^{16}=\frac{1}{2^{9} m_{ \pm}}\left(M_{ \pm}^{2}-\frac{4 m_{ \pm}^{2} \mu^{2}}{[m]^{2}}\right) .
$$

Consistency requires

$$
\frac{1}{m_{+}}\left(M_{+}^{2}-\frac{4 m_{+}^{2} \mu^{2}}{[m]^{2}}\right)=\frac{1}{m_{-}}\left(M_{-}^{2}-\frac{4 m_{-}^{2} \mu^{2}}{[m]^{2}}\right) .
$$

Multiplying by $m_{+} m_{-} /[m]$, setting $x=m_{+} /[m]$ and using $[M]=\mp 2 \mu$ gives

$$
x^{2} \pm \frac{M_{+}}{\mu} x+\frac{M_{+}^{2}}{4 \mu^{2}}=0,
$$

which has the unique solution $x=\mp M_{+} /(2 \mu)$. This then yields $R=0$, which is the singularity. This conclusion can only be avoided if $m_{+}=m_{-}=0$. Therefore a static solution exists if, and only if, the bulk on both sides of the brane is supersymmetric.

The supersymmetric maximal bulk solutions are globally static with a timelike naked singularity at $r_{ \pm}=0$. It is straightforward to see that any static solution describing a single D8-brane has to include these singularities. To avoid the singularities at $r_{ \pm}=0, r_{+}$has to decrease towards the brane on the $(+)$ side and $r_{-}$has to decrease towards it on the $(-)$ side. This means that $\epsilon_{+}=+1$ and $\epsilon_{-}=-1$. However this is the case that was ruled out above. Timelike singularities in dilatonic domain wall spacetimes have been studied in [11].

Bergshoeff et al. gave supersymmetric configurations of parallel branes with vanishing 10-form on both sides of the "stack". This is impossible once the restrictions on $\epsilon$ are taken into account. To see this, start on one side of the configuration, say the left side, with vanishing 10 -form. Let this be the $(+)$ side of the first brane. Then, since $M_{+}=0$ we have $M_{-}=2 \mu$ (if we use a brane rather than an anti-brane) so $Y(R)=\mu^{2}$ and hence $\epsilon_{-}=+1$ so $r_{-}$increases towards the brane on the $(-)$side. This is the $(+)$side of the next brane in the configuration. So for this next brane, $M_{+}=2 \mu$ and $\epsilon_{+}=+1$ (since $r_{+}$ decreases towards this new brane). If we now want $M_{-}=0$ (i.e. vanishing 10-form) on the other side of the brane then $[M]=2 \mu$ so it would actually be an anti-brane. It would have $Y(R)=-\mu^{2}$, which requires $\epsilon_{+}=-1$, a contradiction. (Intuitively this is because we are trying to construct a static brane-antibrane configuration with non-vanishing field strength between them.) Therefore the second brane in the configuration could not be an anti-brane. Hence it must have $M_{-}=4 \mu$, and the argument repeats. One obtains stack of branes with the 10-form field strength increasing as each brane as crossed. Furthermore $R$ decreases as each brane is crossed so one must eventually reach the singularity $R=0$ in finite proper distance.

Note that is is possible to construct a static brane-antibrane configuration but the field strength has to vanish in the region between the brane and anti-brane. So one can have a brane and anti-brane separated by a region of Minkowski space, with the ten-form nonvanishing (and a timelike singularity) on both sides of the configuration. 


\section{B. The case $[m]=0$.}

The equation of motion for the brane simplifies when $m_{+}=m_{-}=m$. This requires $m>0$. Integrating gives

$$
R(\tau)=(17 \sqrt{2 m}|\tau|)^{1 / 17}
$$

and hence

$$
r_{ \pm}(\tau)=(17 \sqrt{2 m}|\tau|)^{26 / 17}
$$

The solutions for positive and negative $\tau$ are related by time reversal so we shall only consider the expanding (positive $\tau$ ) ones. The maximal bulk spacetime has cosmological horizons beyond which $r$ becomes a time coordinate. At large $r$, i.e. late times, the metric can be written

$$
d s^{2} \sim-d \tau^{2}+\tau^{18 / 17} d x^{2}+(17 \sqrt{2 m}|\tau|)^{2 / 17} d \Omega_{0}^{2}
$$

where $x$ is a rescaling of $t$ and we have replaced $r$ with $\tau$. The brane sits at constant $x$ and is comoving with the spatial sections of this anisotropic cosmological solution. The direction transverse to the brane expands faster than directions tangential to it.

Since the RR field is produced by D8 branes, it seems reasonable to assume that $M_{ \pm}$ are integer multiples of the brane charge $\mu$. If we assume that $M_{-}$is an integer multiple of $\mu$ then the solution $\epsilon_{+}=-1, \epsilon_{-}=+1$ is only possible for $M_{-}= \pm \mu$ and hence $M_{+}=\mp \mu$ (as usual the upper sign refers to a brane and the lower sign to an anti-brane). This means that bulk solution is the same on each side of the brane and is symmetric under reflection in the brane. (All of the solutions in [6] are of this form.) The global structure of the maximally extended bulk solution is shown in figure 1. There are two possibilities for the brane trajectory. Let $X_{1}$ denote the region between such a trajectory and the singularity from which it emerges, and $X_{2}$ the complement of this region. To deduce which part of this is relevant when we include the effect of the brane, note that the signs of $\epsilon_{ \pm}$require that $R$ increase towards the brane along its normal. Thus $X_{1}$ is the relevant region. Cutting along the brane and gluing to the mirror image of $X_{1}$ yields the spacetime produced by the brane. It includes the timelike naked singularity from which the brane emerges. Initially signals from the brane can reach the singularity but the presence of a horizon prevents this at late times.

In the non reflection symmetric case we can write $M_{+}= \pm \mu(N-1)$ and $M_{-}= \pm \mu(N+1)$ where $N$ is an integer. Consider first the case $|N|>1$. The solution has $\epsilon_{+}=\epsilon_{-}=\operatorname{sign}(N)$ therefore $R$ must decrease towards the brane on one side and increase towards it on the other so we need a region of type $X_{2}$ on one side of the brane and $X_{1}$ on the other, although the two regions have different values for $M^{2}$ and therefore the brane crosses the horizon in the region with the smaller value of $M^{2}$ before it crosses the horizon in the other region.

The cases $N=1,-1$ are of interest since then the 10 form vanishes on one side of the brane i.e. the bulk on that side is a solution of massless IIA supergravity. In this special case the cosmological horizon vanishes and $r$ is a time coordinate throughout the massless region. Equation 4.37 then holds exactly throughout this region. There is a spacelike singularity at $\tau=0$. The region on the other side of the brane is of type $X_{1}$. 

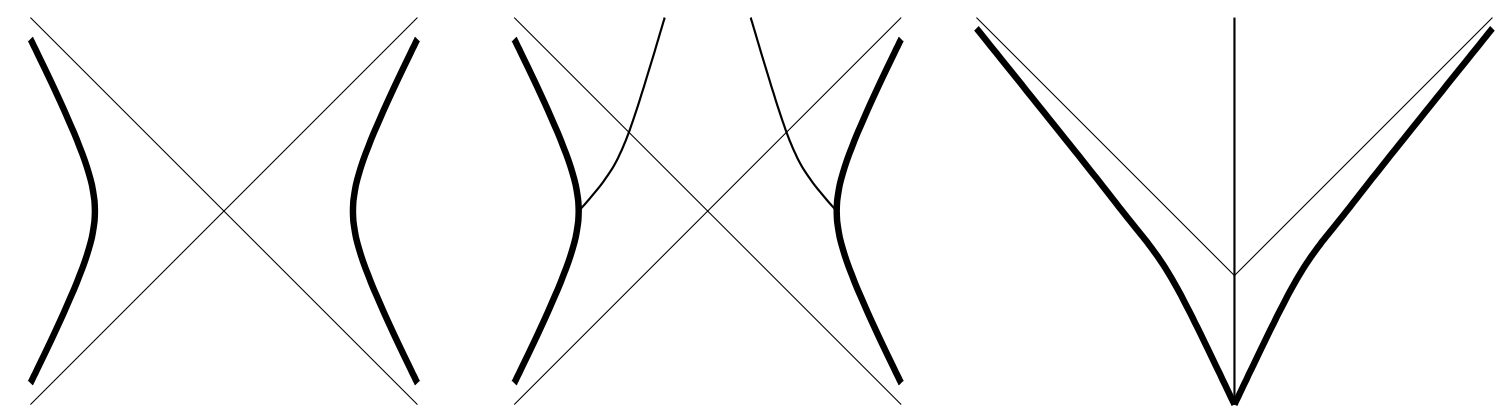

FIG. 1. i) The global structure of the maximally extended bulk spacetime. The thick lines are timelike singularities and the thin lines are cosmological horizons. ii) The possible domain wall trajectories. iii) Global structure of the domain wall spacetime.

\section{The case $[m] \neq 0$.}

When $[m] \neq 0$ it is no longer possible to find $R(\tau)$ explicitly. We shall assume $m_{+}>$ $m_{-}>0$.

Consider first the case $M_{+}=\mp \mu, M_{-}= \pm \mu$. For small $R$, the solution is similar to the $[m]=0$ one. However for larger $R$,

$$
R(\tau) \approx\left(\frac{72}{\mu}[m] \tau\right)^{1 / 9}
$$

At late times, the bulk solution on each side of the brane is of the cosmological form given above i.e. the 8 dimensional spatial sections in the bulk perpendicular have scale factor $\tau^{1 / 17}$. Therefore the brane expands faster than the bulk in these directions. This is because the velocity of the brane in the (spatial) $t$-direction does not tend to zero at large $R$ as it did for the $[m]=0$ case. For small $R, \epsilon_{+}=-1$ and $\epsilon_{-}=+1$ but at the critical value

$$
R^{16}=\frac{\mu^{2}}{2^{7}[m]}
$$

$\epsilon_{+}$changes sign. This occurs in the region where $t_{+}$is a spatial coordinate and corresponds to the brane reaching a turning point in the $t_{+}$-coordinate i.e. changing direction of motion relative to the spatial sections of the bulk spacetime on the $(+)$ side. There is no such turning point on the $(-)$ side. The trajectory of the brane spacetime with respect to the maximal bulk spacetime corresponding to the $(+)$ side of the brane is shown in figure 2 . Considering the signs of $\epsilon_{ \pm}$near $R=0$ shows that it is the region between the brane and the singularity from which it emerges that is relevant on both sides of the brane. This means that the global structure of the resulting brane spacetime is very similar to the $[m]=0$ one.

If we consider a brane with vanishing 10 -form on the $(+)$ side then we have $M_{+}=0$ and $M_{-}=2 \mu$ so

$$
F(R)=-\frac{32}{\mu^{2}}[m]^{2} R^{-16}-m_{+} R^{-32}
$$

The solution for $R(\tau)$ is qualitatively the same as the solution we have just discussed. However we now have $Y(R)>\mu^{2}$ so $\epsilon_{+}=\epsilon_{-}=+1$. On the $(+)$ side of the brane, the 


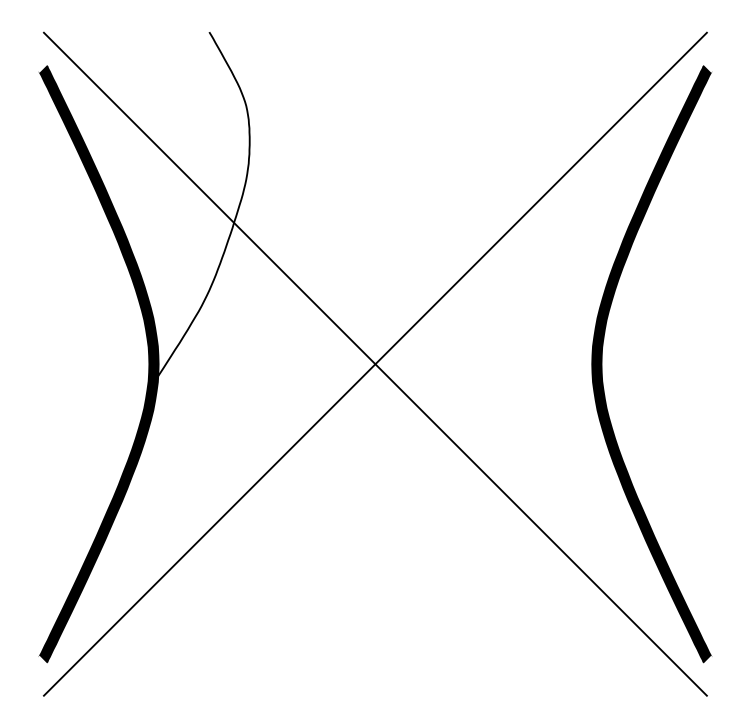

FIG. 2. Trajectory of brane with turning point.

solution is of the cosmological form discussed above and the brane emerges from a spacelike singularity. On the $(-)$ side the relevant region of the maximal bulk spacetime is the region between the brane and the timelike singularity from which it emerges.

Note that for certain choices of the parameters, $F(R)$ is positive for small $R$. This prevents the brane from either emerging from or colliding with the singularity. For such cases, the solutions describe branes that collapse down from infinite $R$ to non-zero $R$ and then re-expand. However the most natural choices for $M$ for a single brane are the symmetric choice $M_{+}=-M_{-}$and the choice for which the 10-form vanishes on one side of the brane. For these choices $F(R)$ is negative everywhere. Positive $F(R)$ presumably corresponds to there being a background 10 -form field present. Since this would have to be produced by other D8 branes it would lead us to dynamic multi-brane configurations, which we shall not discuss here.

\section{THE THEORY OF HOWE, LAMBERT AND WEST}

In the Romans theory the mass arises from a Higgs mechanism whereby the two-form "eats" the vector. However, as Howe, Lambert and West (HLW) [16] have recently pointed out there are actually three distinct Higgs mechanisms, corresponding to whether the vector eats the scalar, the two-form eats the vector or the three-form eats the two-form. In this way, they have constructed a new massive IIA supergravity, which has the appealing property that it can be obtained by compactification of eleven-dimensional Minkowski space on a circle, with the introduction of a Wilson line. In this paper, we will always be assuming that the four-form field strength (coming from the eleven dimensional supergravity bosonic field sector) is turned off. With this caveat, we then obtain the below equations of motion in ten dimensions for the HLW theory:

$$
\begin{aligned}
R_{a b}-\frac{1}{2} g_{a b} R= & -2\left(D_{a} D_{b} \phi-g_{a b} D^{2} \phi+g_{a b}(D \phi)^{2}\right) \\
& +\frac{1}{2}\left(F^{a c} F_{b}^{c}-\frac{1}{4} g_{a b} F^{2}\right) e^{2 \phi}-18 m\left(D_{(a} A_{b)}-g_{a b} D^{c} A_{c}\right)
\end{aligned}
$$




$$
\begin{gathered}
-36 m^{2}\left(A_{a} A_{b}+4 g_{a b} A^{2}\right)-12 m A_{(a} \partial_{b)} \phi \\
-30 m g_{a b} A^{c} \partial_{c} \phi-144 m^{2} g_{a b} e^{-2 \phi}+\text { fermions } \\
D^{b} F_{a b}=18 m A_{b} F_{a}{ }^{b}+72 m^{2} e^{-2 \phi} A_{a}-24 m e^{-2 \phi} \partial_{a} \phi \\
6 D^{2} \phi-8(D \phi)^{2}=-R+\frac{3}{4} e^{2 \phi} F^{2}+360 m^{2} e^{-2 \phi}+288 m^{2} A^{2}+96 m A^{b} \partial_{b} \phi-36 m D^{b} A_{b}
\end{gathered}
$$

where $F_{a b}=\partial_{a} A_{b}-\partial_{b} A_{a}$ as usual. The mass parameter $m$ determines the effective cosmological constant generated by the ten-form field strength. In this paper, we will be simplifying things by truncating these equations of motion by setting the vector field to zero:

$$
A_{(1)}=0
$$

With this assumption, things simplify considerably. Indeed, the only non-trivial remaining equations are the Einstein equation:

$$
R_{a b}=360 m^{2} e^{-2 \phi} g_{a b}
$$

together with the "Maxwell" equation 5.2, which implies that the dilaton $\phi$ is a constant. In other words, if we turn off all of the fields in this theory except for gravity, we simply recover de Sitter spacetime. The effective cosmological constant is then given explicitly in terms of the mass as

$$
\Lambda=1296 m^{2} e^{-2 \phi}
$$

\section{A. 10-form formulation of the HLW theory}

In [16] the authors introduce the exact one form

$$
k=M d y
$$

(In order to avoid annoying numerical factors we have replaced the $m$ of equation 5.4 with $M$, where $M^{2}=m^{2} / 630$.) This vector is both the tangent vector to the eleventh dimension (the $S^{1}$ ) of M-theory, as well as the form used to define a new derivative $D=d+k$. It is natural to dualize this one-form relative to the eleven-dimensional Hodge star operator. When we do this we obtain a ten-form $F_{10}$ which is manifestly covariantly constant:

$$
\star_{11} F_{10}=k
$$

We can use this 10-form to write down a (truncated) action for the HLW theory. Remembering that we are always working only with the sector of the HLW theory where the dilaton is constant, we find that the string frame action takes the form

$$
S=\frac{1}{2 \kappa_{10}^{2}} \int d^{10} x \sqrt{-g}\left(e^{-2 \phi}\left(R+4(\partial \phi)^{2}\right)-\frac{1}{2} e^{-4 \phi} F_{10}{ }^{2}\right)
$$


Clearly, the first thing one notices about this action is the strange factor of $e^{-4 \phi}$ which appears in front of the 10-form. This means that the 10-form of the HLW theory is not a $\mathrm{R}-\mathrm{R}$ sector field and consequently the HLW 8-branes are not D-branes. In fact, this action teaches us something even stranger: If we match dimensions in (5.5) we find that the tension (T) of a brane which couples electrically to $F_{10}$ must scale as

$$
T \sim e^{\phi}
$$

In other words, in the HLW theory the 8-branes have a tension that scales with the 'string coupling'! ? Since we are interested in the gravitational effects of these objects, we are implicitly assuming that $\phi$ is very large in what follows.

Our plan now is simple: The eight-brane divides spacetime up into separate domains, and we use the Israel conditions to match these domains correctly. We now turn our attention to this exercise.

\section{B. Spherical phase transition bubbles in the HLW theory}

By assumption, we are working in the regime of the HLW theory where the dilaton is constant. Consequently, each side of a HLW 8-brane is a portion of a spacetime which is a solution of the vacuum Einstein equations with positive cosmological constant in ten dimensions. In other words, each side of the brane is a portion of de Sitter space, or Schwarzschild-de Sitter space. In general, we can find timelike slices of (Schwarzschild)-de Sitter spacetime which may correspond to the worldvolumes of homogeneous and isotropic 8-branes with arbitrary spatial curvature. This is unlike the case of D8 branes studied above, for which we could only find solutions with Ricci flat spatial sections. We will focus on branes with spherical symmetry

To do this we simply choose a gauge so that the spherically symmetric ten-dimensional metric takes the form [19], [18]:

$$
d s^{2}=-f(r) d t^{2}+\frac{1}{f(r)} d r^{2}+r^{2} d \Omega_{8}^{2}
$$

The most general spherically symmetric vacuum metric in $9+1$ dimensions will have

$$
f(r)=1-\frac{C}{r^{7}}-\frac{\Lambda}{36} r^{2} .
$$

This is of course just the metric of Schwarzschild-de Sitter (SdS) spacetime, where $\Lambda$ is the cosmological constant and $C$ is given in terms of the ADM mass $M$ mass of the black hole as follows:

\footnotetext{
${ }^{7}$ It is worth pointing out here, that since the 3 -form has eaten the 2 -form, there is no field to which F-strings can couple electrically. In this sense, there are no 'strings' in this theory!

${ }^{8}$ The general case was studied in an earlier version of this paper [17], where we found all of the homogeneous and isotropic 8-branes of the HLW theory.
} 


$$
C=\left(\frac{105 G}{16 \pi^{3}}\right) M
$$

Actually, we could also leave $F_{\mu \nu}$ non-zero and consider the resulting Einstein-Maxwelldilaton system of equations; we begin with the $F=0$ truncation for simplicity.

With this simple picture in mind we can now proceed with the equations of motion. The equations of motion for a thin spherical shell, which is bounding a region of SdS from a region of a different SdS spacetime, were worked out for four dimensions in [7]. The analysis developed there will go through here, because the assumption of spherical symmetry means that brane motion is described as a system in $1+1$ dimensions. Put more simply, we are solving for one unknown function, $r(t)$, which describes the radius of the spherical brane at time $t$. We will let $\sigma$ denote the surface energy density of the brane. We will also distinguish between quantities which exist "inside" of the brane, and those which are outside, by using subscripts. Thus, $f_{\text {in }}$ denotes the metric function 5.8 inside the brane, and similarly $f_{\text {out }}$ denotes the same thing outside the brane. The parameters $\epsilon$ have the same interpretation as they had for our D8-brane solutions provided we take the unit normal to point out of the bubble i.e. the interior of the bubble is the $(+)$ region. With all of this in mind, the equation of motion for a spherical bubble bounding two different (vacuum) phases of the HLW supergravity theory is given as

$$
\epsilon_{\text {in }}\left[\dot{r}^{2}+f_{\text {in }}(r)\right]^{1 / 2}-\epsilon_{\text {out }}\left[\dot{r}^{2}+f_{\text {out }}(r)\right]^{1 / 2}=-\frac{\kappa \sigma}{2} r
$$

where $\dot{r}=f^{-1 / 2} \partial_{t} r$ denotes the derivative relative to the time experienced by observers who co-move with the domain wall and $\kappa=8 \pi G$ as always. Any solution of this equation automatically describes an eight-brane worldsheet which satisfies the Israel conditions.

Again, it is easy to see that one can generically rearrange the terms in 5.9 so that the equation of motion for the scale factor is equivalent to the equation of motion for a particle of unit mass and zero total energy moving in a potential $V$. The precise form for $V$ will depend on what one chooses to lie on each side of the 8-brane. Because this is a straightforward generalization of work which has already been done in the setting of four-dimensional cosmology, we will have nothing more to say about these branes here.

\section{Semiclassical instabilities of HLW 8-branes}

The nucleation and annihilation of bubbles of phase transition in the early universe has been studied by a number of authors [20], [8], [7], [22]. Here, we briefly sketch how these results will also go through for HLW branes.

In [22], the authors calculated the probability that one could "create a universe in the laboratory" by quantum tunneling. By this, they meant: What is the probability that a bubble of false vacuum (i.e., de Sitter space) can appear in a lab where we have attained a super-high mass density of the order $10^{76} \mathrm{~g} / \mathrm{cm}^{3}$ ? The authors calculated the rate at which one could create new universes in this way by first finding an instanton, or imaginary time path, which interpolates between the initial state (Schwarzschild) and the final state (Schwarzschild with a bubble of de Sitter in it), then working out the Euclidean action $S_{E}$ for the instanton path and then using the standard semiclassical approximation for the probability $P$ : 


$$
P \propto e^{-S_{E}}
$$

Crudely, they found that the Euclidean action goes like

$$
S_{E} \propto 1 / H^{2}
$$

where $H=\left(\frac{\Lambda}{3}\right)^{1 / 2}$ is the inverse of the Hubble radius. This form of the action follows from the fact that the domain wall sweeps out a three-sphere in imaginary time, and the action is basically the wall tension times the volume of the three-sphere.

In ten dimensions, an HLW 8-brane sweeps out a nine-sphere in imaginary time; it follows that the Euclidean action for the nucleation of HLW branes will go like $1 / H^{8}$. In other words, the nucleation of isolated bubbles of massive phase in this IIA supergravity theory will still be highly suppressed.

This shows that HLW branes can be spontaneously nucleated, but what about the time reverse: How do they annihilate? In a recent paper, Kolitch and Eardley [20] studied the decay of Vilenkin-Ipser-Sikivie (VIS) 21] domain walls in cosmology. These VIS domain walls are the Minkowski-Minkowski version of the de Sitter-de Sitter (dS-dS) HLW branes discussed above. That is to say, a VIS domain wall is a repulsive spherical bubble which separates two (compact) portions of Minkowski space (just as a dS-dS HLW brane separates two compact portions of de Sitter spacetime). It is not hard to see that their construction will in fact go through for the dS-dS HLW branes. Again, the action for the instanton describing such an annihilation event will go crudely as $1 / H^{8}$.

It is clear that black hole pairs will be nucleated in the presence of the repulsive, dS-dS spherical HLW branes. This is basically because these dS-dS branes are bubbles bounding two regions of inflationary phase, and we know that black holes will be produced in an inflating (or domain wall [23]) background. Of course, just about anything can be produced in an inflationary background, simply because the repulsive gravitational energy is a natural source capable of pulling virtual loops of matter out of the vacuum. In particular, it is well known that topological defects [24 will also be nucleated in inflation. Typically, a defect nucleates at the Hubble radius $r=H^{-1}$. If the defect is much thinner than the scale of the universe at the moment of nucleation, it makes sense to model the defect using the Nambu action. That is, it makes sense to assume that the defect is "infinitely thin", and to define the action to be the area of the worldvolume swept out by the defect (multiplied by the characteristic tension, or mass, of the defect). In this limit, the instanton for a loop of string is a two-sphere of radius $H^{-1}$. Similarly, the instanton for a (closed) spherical domain wall is a three-sphere of radius $H^{-1}$, and so on. Thus, the Euclidean action for defect nucleation generically has the form

$$
S_{E}=\mu \operatorname{Vol}\left(S^{n}(1 / H)\right)
$$

where $\operatorname{Vol}\left(S^{n}(1 / H)\right)$ denotes the volume of an n-sphere of radius $1 / H$, and $\mu$ denotes the mass of the monopoles (if $n=1$ ), the tension of the string loop (if $n=2$ ) or the energy density of the domain wall (if $n=3$ ). One therefore finds that the rate of production of these defects is strongly suppressed if the defect tension is very large, or the cosmological constant is very small, as would be expected. Similarly, light defects are likely to be produced in a background with a large cosmological constant. 


\section{CONCLUSION}

We have found solutions of the coupled bulk-worldvolume theory which describe the motion of a D8-brane in massive IIA supergravity. Since the dilaton is running in any such background, it follows that the energy-momentum carried by that field is allowed to move from the brane to the bulk and vice-versa. We have also shown that whenever a D8-brane starts to move, a non-extremality term will necessarily appear in the bulk on both sides of the brane. This term obstructs the existence of Killing spinors and therefore breaks any supersymmetry which was initially present. The spacetime on either side of the brane at late times is typically an anisotropic cosmological solution with the direction transverse to the brane expanding faster than the directions tangential to it. When the non-extremality parameter is the same on both sides of the brane the brane eventually becomes comoving with the bulk. If the non-extremality parameters are not the same then the brane always moves relative to the bulk.

Use of the Israel conditions also places constraints on supersymmetric solutions. We have shown that it is not possible to exclude the singularity from a supersymmetric D8-brane spacetime (unless one introduces objects of negative tension, such as orientifold planes). We have also shown that it is impossible to construct a configuration of parallel D8 branes (or anti-branes) that is a solution of the massless IIA theory in the two asymptotic regions.

We have also discussed branes in the Howe-Lambert-West theory. Unlike our D8 brane solutions, these can have spatial sections with Ricci curvature, and such spherical branes might be relevant for the study of phase transitions in the supergravity theory. It would be interesting to see if spherical D8 brane solutions could be found, although this would probably have to be done numerically. If such solutions exist then they could describe the evolution of a bubble of the massive phase of IIA supergravity surrounded by the massless phase.

Perhaps the most exciting thing which these results teach us is that it is possible to describe gravitating brane configurations without losing sight of the brane worldvolume. To put it another way, there is nothing to stop one from defining an effective worldvolume action for these gravitating D8-branes (presumably the Born-Infeld-Dirac action will do), and studying these branes from the worldvolume point of view. Again, since the dilaton will flow into the bulk, there will be an anomaly in the stress-energy conservation equation for the worldvolume theory. It would be amusing to see how this is related to the breakdown of supersymmetry.

This should be contrasted to other heavy, or gravitating, brane configurations in supergravity theories, such as the non-dilatonic p-branes 25. There, the brane worldvolume vanishes, leaving a geometry which looks rather like a black hole (extended in some extra dimensions), such that the solution interpolates between different vacua (generically Minkowski spacetime far from the brane, and $(a d S)_{p+2} \times S^{D-p-2}$ near the brane [25]). In these solutions, there is no brane to be found and it is therefore meaningless to talk about worldvolume actions.

The results [4. 5 concerning how a string ending on a D-brane will tug on the brane beg the question: How will a heavy string tug on a heavy D8-brane? One has to be careful in posing this question, since it is not at all clear that the method of assigning distributional curvatures to spacetimes of low differentiability (i.e. imposing the Israel conditions) will 
still make sense when one is dealing with extended objects of codimension greater than one. Research into these problems is currently underway.

\section{Acknowledgements}

The authors would like to thank Gary Gibbons, Mike Green, Julius Kuti, and Neil Lambert for useful conversations. A.C. is supported by a Drapers Research Fellowship at Pembroke College, Cambridge. M.J.P. was partially supported by a grant from the U.S. Department of Energy. 


\section{REFERENCES}

[1] J. Polchinski, Dirichlet-Branes and Ramond-Ramond Charges, Phys. Rev. Lett. 75, 4724 (1995); hep-th/9510017.

[2] J. Dai, R.G. Leigh and J. Polchinski, New Connections between String Theories, Mod. Phys. Lett. A4, 2073 (1989).

[3] L. Thorlacius, Introduction to D-branes, Nucl. Phys. Proc. Suppl. 61A: 86-98, (1998); hep-th/9708078.

[4] G.W. Gibbons, Born-Infeld particles and Dirichlet p-branes, Nucl. Phys. B514: 603639, (1998); hep-th/9709027.

[5] Curtis G. Callan, Jr. and J.M. Maldacena, Brane death and dynamics from the BornInfeld action, Nucl. Phys. B513: 198-212, (1998); hep-th/9708147.

[6] A. Chamblin and H.S. Reall, Dynamic Dilatonic Domain Walls, hep-th/9903225, submitted to Nucl. Phys. B.

[7] V.A. Berezin, V.A. Kuzmin, and I.I. Tkachev, Dynamics of bubbles in general relativity, Phys. Rev. D 36, No. 10, 2919-2944 (1987).

[8] Steven K. Blau, E.I. Guendelman, and Alan H. Guth, Dynamics of false-vacuum bubbles, Phys. Rev. D 35, No. 6, 1747-1766 (1987).

[9] W. Israel, Nuovo Cimento 44B, 1 (1966); 48B, 463E (1967).

[10] M. Cvetic, S. Griffies and H.H. Soleng, Local and global gravitational aspects of domain wall space-times, Phys. Rev. D 48, 2613-2634 (1993); gr-qc/9306005.

[11] N. Cvetic and H.H. Soleng, Naked singularities in dilatonic domain wall space-times, Phys. Rev. D51, 5768-5784 (1995); hep-th/9411170.

[12] M. Cvetic and H.H. Soleng, Supergravity domain walls, Physics Reports 282, 159-223 (1997), hep-th/9604090.

[13] L.J. Romans, Massive $N=2$ a supergravity in ten dimensions, Phys. Lett. 169B, No. 4, 374-380 (1986).

[14] E. Bergshoeff, M. de Roo, M.B. Green, G. Papadopoulos and P.K. Townsend, Duality of Type II 7-branes and 8-branes, Nucl. Phys. B470, 113-135 (1996); hep-th/9601150.

[15] D. Brecher and M.J. Perry, Ricci-Flat Branes, hep-th/9908018.

[16] P.S. Howe, N.D. Lambert and P.C. West, A new massive Type IIA supergravity from compactification, Phys. Lett. B416: 303-308, (1998); hep-th/9707139 v2.

[17] A. Chamblin and M.J. Perry, hep-th/9712112.

[18] R.C. Myers and M.J. Perry, Black holes in higher dimensional space-times, Annals of Physics 172, 304-347 (1986).

[19] F.R. Tangherlini, Schwarzschild field in $n$ dimensions and the dimensionality of space problem, Il Nuovo Cimento, Vol. XXVII, 636-651, (1963).

[20] Shawn J. Kolitch and Douglas M. Eardley, Quantum decay of domain walls in cosmology: 1. Instanton approach, Phys. Rev. D 56: 4651-4662, (1997); gr-qc/9706011.

[21] A. Vilenkin, Phys. Lett. B133, 177-179 (1983); J. Ipser and P. Sikivie, Phys. Rev. D 30, 712-719 (1984).

[22] E. Farhi, A.H. Guth and J. Guven, Nucl. Phys. B339, 417 (1990).

[23] R. Caldwell, A. Chamblin and G.W. Gibbons, Pair creation of black holes by domain walls, Phys. Rev. D 53, 7103-7114 (1996), hep-th/9602126; A. Chamblin and J.M.A. Ashbourn-Chamblin, Black hole pairs and supergravity domain walls, Phys. Rev. D 57: 3529-3536, (1998); hep-th/9612014. 
[24] A. Vilenkin and E.P.S. Shellard, Cosmic Strings and Other Topological Defects, Cambridge University Press, Cambridge (1994).

[25] G.W. Gibbons, P.K. Townsend, Vacuum interpolation in supergravity via super p-branes, Phys. Rev. Lett. 71, 3754-3757, (1993); hep-th/9307049. 